\title{
Caracterização reológica de suspensões cimentícias mistas com cales ou filitos
}

\author{
Rheological characterization of cementitious suspensions \\ mixed with lime or phyllites
}

\begin{tabular}{|c|c|}
\hline & $\begin{array}{l}\text { Roberto Cesar de Oliveira Romano } \\
\text { Mauro Adamo Seabra } \\
\text { Vanderley Moacyr John } \\
\text { Rafael Giuliano Pileggi }\end{array}$ \\
\hline $\begin{array}{r}\text { Roberto Cesar de Oliveira } \\
\text { Romano } \\
\text { Universidade de São Paulo } \\
\text { São Paulo - SP - Brasil }\end{array}$ & 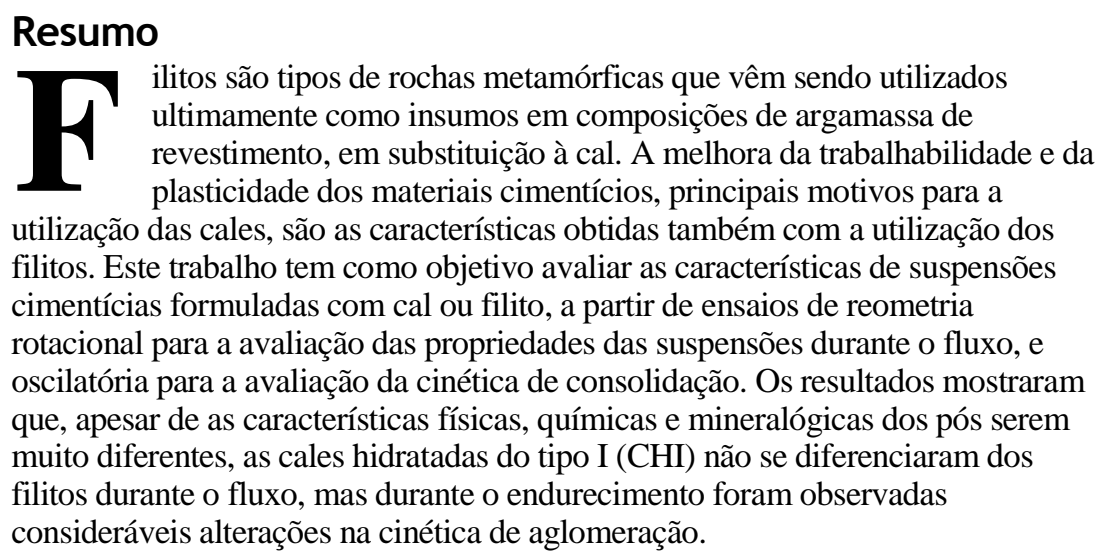 \\
\hline & Palavras-chave: Reologia. Cal. Filito. Blenda. \\
\hline & Abstract \\
\hline São Paulo - SP - Brasil & $\begin{array}{l}\text { Phyllites are a type of metamorphic rock that is now being used as raw material in } \\
\text { the composition of rendering mortar, replacing lime. The improvement on the }\end{array}$ \\
\hline $\begin{array}{l}\text { Vanderley Moacyr John } \\
\text { Universidade de São Paulo } \\
\text { São Paulo - SP - Brasil }\end{array}$ & $\begin{array}{l}\text { workability and plasticity of cementitious materials, the main reasons for the use } \\
\text { of limes, are features that are also obtained with the use of phyllites. The aim of } \\
\text { this study is to evaluate the characteristics of cementitious suspensions formulated } \\
\text { with lime or phyllite through the use of rotational rheometry tests to evaluate the }\end{array}$ \\
\hline $\begin{array}{l}\text { Rafael Giuliano Pileggi } \\
\text { Universidade de São Paulo } \\
\text { São Paulo - SP - Brasil }\end{array}$ & $\begin{array}{l}\text { suspensions' properties during the flow, and oscillatory rheometry to evaluate } \\
\text { kinetic consolidation. The results showed that although the physical, chemical and } \\
\text { mineralogical properties of the powders are very different, the type I hydrated } \\
\text { limes (CHI) were not different from the phyllites during the flow, but during the }\end{array}$ \\
\hline Rece & consolidation the CHI limes had a faster kinetic agglomeration than the phyllites. \\
\hline Ace & ds: Rheology. Lime. Phyllite. Blend. \\
\hline
\end{tabular}

ROMANO, R. C. DE O.; SEABRA, M. A.; JOHN, V. M.; PILEGGI, R. G. Caracterização reológica de suspensões cimentícias 75 mistas com cales ou filitos. Ambiente Construído, Porto Alegre, v. 14, n. 1, p. 75-84, jan./mar. 2014. ISSN 1678-8621 Associação Nacional de Tecnologia do Ambiente Construído. 


\section{Introdução}

O uso de filitos como substitutos da cal em formulações de argamassas de revestimento ou assentamento tem sido comum no setor construtivo, por conferirem às composições cimentícias, no estado fresco, características similares às obtidas com o uso do ligante aéreo (JOHN, 2003; ROMANO et al., 2011).

Trata-se de materiais que não apresentam características ligantes, diferentemente das cales de construção (QUARCIONI; CINCOTTO, 2008), ficando a consolidação e o endurecimento de tais composições somente a cargo da ação do cimento. Por isso, os fabricantes os comercializam, em muitos casos, misturados com cales, o que foi chamado neste trabalho de blenda.

Apesar de o controle de qualidade das cales ser embasado em normalização técnica, esse tipo de insumo somente é qualificado em função das características físicas, como distribuição granulométrica, densidade e área superficial específica, ou em função da composição química, perda ao fogo e resíduo insolúvel.

Por outro lado, apesar de a presença dos filitos, ou das cales, melhorar as propriedades das argamassas no estado fresco, poucos trabalhos são reportados em literatura com foco em caracterizações reológicas, com ênfase no fluxo ou durante a consolidação (ROMANO et al., 2013).

Dessa forma, este trabalho foi realizado com o objetivo de avaliar as características de pastas cimentícias formuladas com cal ou filito durante o fluxo ou na transição de comportamento de fluido viscoso para sólido elástico, a partir de reometria rotacional e oscilatória.

\section{Revisão da literatura}

\section{Avaliação do perfil reológico das suspensões por reometria rotacional}

O princípio de reometria rotacional consiste na aplicação de uma tensão ou de uma taxa de cisalhamento a uma amostra e aquisição, como resposta, da resistência do fluido ao fluxo viscosidade - e da tensão mínima necessária para o início do fluxo - tensão de escoamento.

Pode-se, ainda, obter informações sobre o comportamento reológico do fluido (newtoniano, Bingham, pseudoplástico, dilatante) e do perfil de tixotropia, informações importantes para a correta compreensão das características dos materiais durante o transporte, bombeamento ou aplicação.
Para se utilizar tal técnica de medição, é necessário, inicialmente, entender que tipo de informação se pretende extrair de cada fluido. Para tanto, os sistemas mais utilizados são os cilindros coaxiais (com geometria do tipo Vane ou DIN), geometrias do tipo cone-placa e placas paralelas (geometria de ensaio escolhida para a realização deste trabalho).

Em cada caso, há uma forma de cálculo da viscosidade $(\mu)$ ou da tensão $(\tau)$, dependente do tipo de sistema adotado, que, para a geometria de placas paralelas, são apresentadas nas Equações 1 a 3:

Taxa de cisalhamento $\dot{\gamma}=\frac{\omega R}{h}$

Eq. 1

Viscosidade $\mu=\frac{T .2 h}{\pi \omega R^{4}}$

Eq. 2

Tensão de cisalhamento $\tau=\frac{2 T}{\pi R^{3}}$

Eq. 3

Onde:

$\gamma$ 'é a taxa de cisalhamento;

$\omega$ é a velocidade angular;

$\mathrm{R}$ é o raio da geometria;

h é a espessura da amostra (gap de ensaio); e

$\mathrm{T}$ é o torque de cisalhamento.

Em fluidos com perfil reológico do tipo newtoniano, a viscosidade não sofre influência de $\gamma$ : Fluidos pseudoplásticos apresentam diminuição da viscosidade aparente com o aumento da taxa, enquanto fluidos dilatantes são caracterizados pelo aumento da viscosidade em função do aumento da taxa.

\section{Acompanhamento da cinética de aglomeração por reometria oscilatória}

Os fenômenos que induzem a aglomeração nos sistemas cimentícios podem ser descritos como a contribuição física do processo de consolidação; por isso, é essencial a avaliação da magnitude das forças atrativas em função do tempo (FLATT, 2004; ROUSSEL et al., 2010; ROMANO et al., 2013).

Ensaios oscilatórios com frequência e deformação controladas (time sweep) são os mais indicados para o monitoramento da aglomeração em função do tempo, desde que sejam realizados dentro da região viscoelástica linear (RVL) da suspensão, onde a deformação imposta durante o ensaio está abaixo da deformação crítica da pasta cimentícia. Usualmente para esse tipo de suspensão são

76 Romano, R. C. de O.; Seabra, M. A.; John, V. M.; Pileggi, R. G. 
utilizados valores na faixa de $10^{-4}$. Nessa condição a estrutura formada continuamente, devido às alterações na estrutura do cimento, não é rompida durante o teste.

O ensaio oscilatório consiste na aplicação de uma deformação senoidal $(\gamma)$ à amostra, e como resposta se obtém a tensão, de acordo com as Equações 4 e 5, onde $\omega$ é a velocidade angular, t é o tempo, $\gamma_{0}$ é a amplitude máxima de deformação, $\tau_{0}$ é a tensão de escoamento e $\delta$ é a fase do ângulo (ROMANO et al., 2013).

$$
\begin{array}{ll}
\gamma=\gamma_{0} \cdot \operatorname{sen} \omega t & \text { Eq. } 4 \\
\tau=\tau_{0} \cdot \operatorname{sen}(\omega t+\delta) & \text { Eq. } 5
\end{array}
$$

Idealmente, quando a tensão e a deformação estão em fase $(\delta=0)$, o material se comporta tipicamente como um sólido hookeano (elástico), enquanto, se a tensão e a deformação estão fora de fase $\left(\delta=90^{\circ}\right)$, o material apresenta comportamento típico de fluido newtoniano (viscoso). No entanto, os materiais reais não se comportam puramente como sólidos ou como fluidos durante a consolidação, apresentando um comportamento intermediário $\left(0<\delta<90^{\circ}\right)$ conhecido como viscoelástico.

A partir das Equações 4 e 5 pode ser calculado o módulo complexo $\left(\mathrm{G}^{*}\right)$ em função da relação entre $\gamma_{0}$ e $\tau_{0}$, conforme ilustrado na Equação 6:

$G^{*}=\frac{\tau_{0}}{\gamma_{0}}$

Eq. 6

Como $\mathrm{G}^{*}$ é um número complexo, pode ser dividido em duas componentes: uma real ( $\mathrm{G}^{\prime}$ módulo de armazenamento elástico) e outra imaginária (G", módulo de perda viscosa), de acordo com as Equações 7 e 8:

$G^{\prime}=G^{*} \cdot \cos \delta$

Eq. 7

$G^{\prime \prime}=G^{*} \cdot \operatorname{sen} \delta$

Eq. 8

Como a microestrutura formada continuamente não é rompida durante o ensaio realizado dentro do limite viscoelástico linear, os resultados de G' obtidos como resposta apresentam equivalência com os resultados de tensão de escoamento. Sendo assim, a $\tau_{0}\left(\equiv \mathrm{G}^{\prime}\right)$ pode ser avaliada continuamente ao longo do tempo.

\section{Materiais utilizados}

Os materiais utilizados neste trabalho foram divididos em três tipos: cal $(\mathrm{C})$, filito $(\mathrm{F})$ ou blenda ( $\mathrm{B}$, misturas de cal e filito). Como foram utilizados diferentes cales e filitos, as amostras foram separadas por números, visando manter em sigilo o nome dos fabricantes. No caso das cales, $\mathrm{C} 1$ e $\mathrm{C} 2$ são do tipo CHI, e C3 é do tipo CHIII.
Métodos de ensaio

\section{Caracterização química via seca}

A fluorescência de raios $\mathrm{X}$ foi realizada nas amostras fundidas, em um espectrômetro Pan Panalytical Magix-Pro. Adicionalmente foi realizada a medida de perda ao fogo $(\mathrm{PF})$ e de resíduo insolúvel (RI).

\section{Distribuição granulométrica a laser}

Os ensaios foram efetuados em equipamento da marca Malvem, modelo Mastersizer S long bed Ver 2.19, com faixa de detecção de $0,05 \mu \mathrm{m}$ a 3,50 $\mathrm{mm}$.

\section{Densidade real}

Foi determinada por picnometria por adsorção de gás $\mathrm{He}$ em um equipamento Multipinometer, da marca Quantachrome MVP 5DC, a partir de uma média de 5 determinações de cada matéria-prima.

\section{Área superficial específica}

Foi utilizado o método de adsorção de gás N2, conhecido como BET (desenvolvida por Braunauer, Emmet e Teller), em um equipamento Micromeritics ASAP 2010, após tratamento das amostras sob aquecimento e vácuo.

\section{Reometria rotacional}

Um volume de $2 \mathrm{~mL}$ de pasta foi colocado no reômetro (AR550, TA Instruments) e iniciou-se o ensaio de fluxo contínuo, 12 min após o início da mistura. Na primeira etapa do ensaio (aceleração), a taxa de cisalhamento foi aumentada partindo-se do repouso até $150 \mathrm{~s}^{-1} \mathrm{e}$, em seguida, foi reduzida, retornando-se para o repouso (desaceleração). O ciclo de aceleração/desaceleração foi realizado em um tempo de 2 min.

\section{Reometria oscilatória}

Após o ensaio de fluxo, a amostra foi solicitada por oscilação com controle de frequência $(1 \mathrm{~Hz})$ e deformação $\left(10^{-4}\right)$ por $4 \mathrm{~h}$, a fim de se avaliar a consolidação dos materiais.

\section{Resultados}

Vários são os fatores que interferem nas propriedades reológicas das pastas cimentícias, entre os quais podem ser citados teor de água, área superficial específica, densidade, distribuição granulométrica. Por isso, como os materiais utilizados no trabalho são muito heterogêneos, é de 
extrema importância o estudo completo das matérias-primas constituintes das pastas, desde a caracterização das partículas até a consolidação dos materiais.

\section{Caracterização Química}

A composição química das matérias-primas é apresentada na Tabela 1, obtidas a partir de espectroscopia de fluorescência de raios X.

As cales são constituídas principalmente por óxidos e hidróxidos de cálcio e magnésio, com pequena quantidade de resíduo insolúvel. Os filitos, por outro lado, são materiais constituídos de minerais, possuem elevados teores de sílica e óxido de alumínio, sendo a quantidade de $\mathrm{CaO}$ e $\mathrm{MgO}$ inferior a $2 \%$, o que ilustra claramente a falta de material ligante.

De acordo com as quantidades de $\mathrm{MgO}$ e $\mathrm{CaO}$, pode-se dizer que as cales C1 e C3 são provenientes de calcários dolomíticos e que a cal C2 é proveniente de calcário calcítico.

Nos casos das blendas B1, B2 e B3, foram utilizadas cales calcíticas na mistura com o filito, já que essas apresentam maiores quantidades de $\mathrm{CaO}$.

A elevada quantidade de resíduo insolúvel e a menor perda ao fogo observadas nos filitos decorrem da maior quantidade de material carbonático presente na composição. Por ser uma mistura, as blendas sempre apresentaram valores intermediários entre as cales e os filitos.

Sendo assim, é possível concluir que nenhum dos filitos ou blendas se apresenta em total conformidade às especificações normativas aplicadas para as cales, não sendo possível afirmar que, quimicamente, sejam compatíveis com as cales. Por isso não podem ser classificados como tais ligantes.

\section{Caracterização física e granulométrica}

As distribuições granulométricas das matériasprimas são ilustradas na Figura 1. Em (a) são apresentados os resultados para as cales, em (b) para as blendas e em (c) para os filitos.

A cal C2 apresentou uma estreita distribuição e tamanho de partículas com diâmetro máximo de 40 $\mu \mathrm{m}$ (dentro do esperado para o ligante), enquanto as demais cales apresentaram distribuição mais aberta, com partículas de até $200 \mu \mathrm{m}$. Para os filitos e blendas, a distribuição granulométrica foi também aberta, com algumas distribuições bimodais, indicando pequeno beneficiamento aplicado aos materiais durante sua produção (JOHN, 2003).

A densidade real dos pós, a massa unitária conforme realizado em obra (método gravimétrico) e a área superficial específica das matérias-primas são apresentadas na Tabela 2.

Os filitos apresentaram maiores densidades reais que as cales e as blendas, e, conforme previsto, a área superficial específica (ASE) das cales foi maior que para as demais matérias-primas. $\mathrm{O}$ que chama a atenção é a elevada ASE do filito F1, muito diferente dos demais filitos e superior a todas as blendas, o que pode influenciar nas características das pastas durante o fluxo.

Juntamente com a distribuição de tamanho de partículas, a área superficial específica influencia diretamente as propriedades reológicas, seja durante o fluxo ou endurecimento, pois impactam diretamente na distância de separação entre as partículas na pasta. $\mathrm{O}$ teor de água utilizado para o processamento deve ser suficiente para exceder a porosidade de empacotamento e recobrir a superfície das partículas, gerando uma distância de separação mínima de $50 \mathrm{~nm}$ entre elas, podendo, assim, prever uma boa fluidez na pasta (OLIVEIRA et al., 2000).

Tabela 1 - Proporções volumétricas dos componentes das matérias-primas

\begin{tabular}{c|cccccccc}
\hline Matéria-prima & $\mathbf{S i O}_{\mathbf{2}}$ & $\mathbf{A l}_{\mathbf{2}} \mathbf{O}_{\mathbf{3}}$ & $\mathbf{F e}_{\mathbf{2}} \mathbf{O}_{\mathbf{3}}$ & $\mathbf{M g O}$ & $\mathbf{C a O}$ & Outros & $\mathbf{P F}$ & $\mathbf{R I}$ \\
\hline C1 & 1,62 & 0,66 & 0,25 & 27,90 & 41,50 & 0,21 & 28,30 & 1,9 \\
C2 & 0,89 & 0,22 & 0,24 & 0,37 & 72,40 & 0,18 & 23,30 & 6,6 \\
C3 & 4,03 & 0,40 & 0,20 & 28,10 & 39,50 & 0,21 & 28,20 & 4,8 \\
\hline B1 & 23,20 & 2,13 & 0,15 & 13,40 & 35,20 & 0,44 & 25,50 & 30,2 \\
B2 & 41,90 & 12,70 & 1,15 & 1,12 & 24,60 & 3,36 & 15,30 & 54,4 \\
B3 & 22,30 & 5,80 & 0,80 & 2,05 & 42,80 & 1,82 & 21,60 & 26,7 \\
B4 & 58,90 & 10,80 & 2,08 & 7,05 & 8,78 & 3,02 & 9,73 & 72,7 \\
\hline F1 & 68,80 & 17,10 & 4,65 & 0,82 & 0,10 & 4,54 & 4,95 & 92,0 \\
F2 & 74,00 & 14,70 & 2,21 & 1,03 & 0,09 & 5,89 & 2,97 & 92,6 \\
F3 & 60,50 & 18,20 & 9,92 & 1,58 & 0,21 & 5,65 & 4,38 & 92,8 \\
\hline
\end{tabular}

Nota: * soma das quantidades de $\mathrm{MnO}, \mathrm{Na}_{2} \mathrm{O}, \mathrm{K}_{2} \mathrm{O}, \mathrm{TiO}_{2}$ e $\mathrm{P}_{2} \mathrm{O}_{5}$. 
Figura 1 - Distribuições granulométricas das (a) cales, (b) das blendas e (c) dos filitos
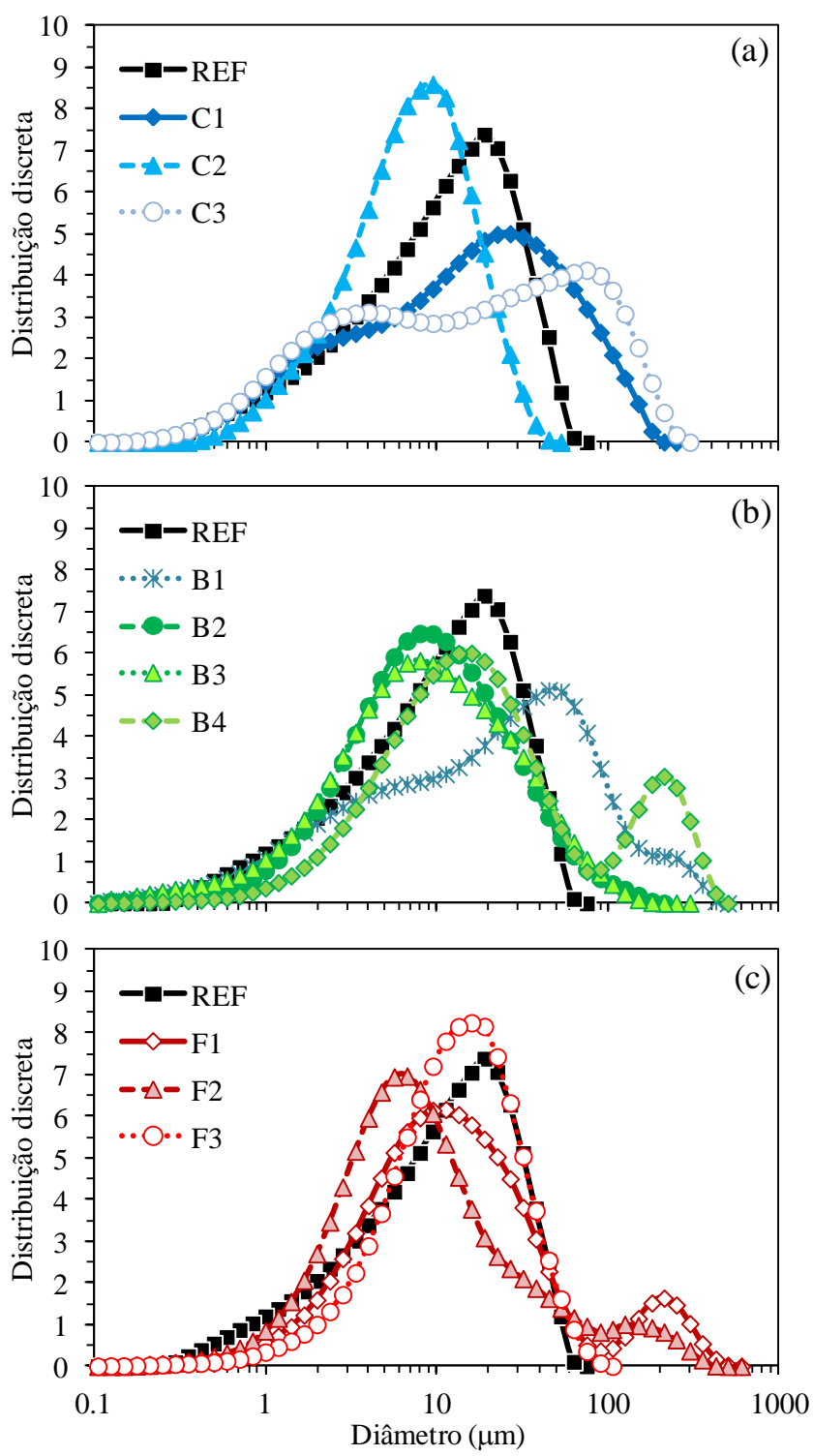

Tabela 2 - Características físicas das matérias-primas

\begin{tabular}{c|c|c|c}
\hline Matéria-prima & $\begin{array}{c}\text { Densidade real } \\
\left(\mathbf{g} / \mathbf{c m}^{\mathbf{3}}\right)\end{array}$ & $\begin{array}{c}\mathbf{M U} \\
\left(\mathbf{g} / \mathbf{c m}^{\mathbf{3}}\right)\end{array}$ & $\begin{array}{c}\mathbf{A S E} \\
\left(\mathbf{m}^{\mathbf{2}} \mathbf{g}\right)\end{array}$ \\
\hline REF & 3,00 & 1,06 & 1,75 \\
\hline C1 & 2,45 & 0,72 & 15,90 \\
C2 & 2,62 & 0,49 & 14,50 \\
C3 & 2,60 & 0,76 & 10,60 \\
\hline B1 & 2,70 & 0,81 & 8,50 \\
B2 & 2,60 & 0,79 & 8,80 \\
B3 & 2,47 & 0,62 & 6,00 \\
B4 & 2,67 & 0,84 & 4,40 \\
\hline F1 & 2,80 & 0,87 & 13,70 \\
F2 & 2,76 & 0,79 & 4,20 \\
F3 & 2,90 & 0,89 & 3,20 \\
\hline
\end{tabular}

Nota: Legenda:

MU - massa unitária; e

ASE - área superficial específica. 


\section{Proporções das matérias-primas utilizadas nas pastas}

As pastas cimentícias foram processadas com uma proporção entre as matérias-primas de 1:1, em volume (calculada a partir da massa unitária das matérias-primas), e relação água/materiais secos de $0,40\left(\mathrm{~V}_{\text {água }} / \mathrm{V}_{\mathrm{pó}}\right)$. Tal estratégia foi adotada para melhor representar o que poderia ocorrer nos canteiros de obra, onde as matérias-primas são dosadas em volume.

As massas dos pós e da água pesados para a mistura das pastas estão apresentadas na Tabela 3. Em todos os casos foi utilizado o cimento CPIIF da marca Ribeirão.

\section{Procedimento de mistura}

Inicialmente, homogeneizaram-se os pós secos; em seguida, todo o pó foi adicionado na água e misturou-se a pasta por $2 \mathrm{~min}$ em $500 \mathrm{rpm}$, em um misturador de bancada RW 20 (IKA, Labortechnik). Aumentou-se a rotação para 1.000 rpm por mais 2 min e, em seguida, para $1.200 \mathrm{rpm}$ por mais $2 \mathrm{~min}$.

\section{Caracterização reológica: reometria rotacional}

As características reológicas de pastas cimentícias estão diretamente relacionadas com o teor de água, tempo e temperatura, composição e finura do cimento e dos demais componentes da mistura, energia de mistura, propriedades físicas e químicas das partículas, etc. (BANFILL, 2007; ROMANO et al., 2013).

Neste trabalho, o tipo de cimento, a temperatura, o teor de água e a forma de processamento foram mantidos constantes e, por isso, pode-se dizer que as alterações geradas nas propriedades das pastas ocorreram devido às variações das características físicas e químicas dos pós utilizados na formulação.

A Figura 2 ilustra as alterações na viscosidade dinâmica (a) e na tensão de cisalhamento (b) em função da taxa de cisalhamento aplicada no ensaio. Acima estão os resultados das pastas com as cales, no meio das pastas com as blendas e abaixo com os filitos. Em destaque são apresentados os resultados da área de histerese (área inscrita entre as curvas de aceleração e desaceleração) (LIBERATO et al., 2013).

Todas as pastas avaliadas apresentam comportamento de fluidos pseudoplásticos com tensão de escoamento. No entanto, as áreas de histerese são muito distintas, ilustrando as peculiaridades de cada cal ou argilomineral estudado.

Com exceção da pasta referência, misturada somente com cimento, em todas as pastas mistas as partículas se encontravam aglomeradas no início do ensaio de fluxo (baixa taxa de cisalhamento), devido à intensificação das forças de origem superficiais agindo sobre elas, favorecendo as interações de van der Waals e as forças de capilaridade. Tal intensificação ocorreu principalmente devido ao aumento da área superficial específica do pó, aliada à diminuição da distância de separação entre as partículas na pasta (OLIVEIRA et al., 2000).

Com o aumento da taxa de cisalhamento, esses aglomerados foram rompidos, liberando a água que se encontrava aprisionada em seu interior, o que resultou em diminuição da tensão de cisalhamento em função do aumento da taxa de cisalhamento até o máximo valor aplicado. Nesta primeira etapa do ensaio (aceleração) ocorreu, então, a desaglomeração das partículas.

Tabela 3 - Massas das matérias-primas utilizadas nas pastas

\begin{tabular}{l|c|c|c|c}
\hline \multicolumn{2}{c|}{ Matéria-prima } & Amostra (g) & Cimento (g) & Água (g) \\
\hline Cimento & REF & - & 47,17 & 18,87 \\
\hline \multirow{3}{*}{ Cales } & C1 & 20,22 & 29,78 & 22,47 \\
& C2 & 15,81 & 34,19 & 25,81 \\
& C3 & 20,88 & 29,12 & 21,98 \\
\hline \multirow{4}{*}{ Blendas } & B1 & 21,66 & 28,34 & 21,39 \\
& B2 & 21,35 & 28,65 & 21,62 \\
& B3 & 18,45 & 31,55 & 23,81 \\
& B4 & 22,11 & 27,89 & 21,05 \\
\hline \multirow{4}{*}{ Filitos } & F1 & 22,54 & 27,46 & 20,73 \\
& F2 & 21,35 & 28,65 & 21,62 \\
& F3 & 22,82 & 27,18 & 20,51 \\
\hline
\end{tabular}

80 Romano, R. C. de O.; Seabra, M. A.; John, V. M.; Pileggi, R. G. 
Figura 2 - (a) Viscosidade e (b) tensão de cisalhamento das pastas em função da taxa de cisalhamento. Acima estão os resultados das pastas com as cales; no meio, das pastas com as blendas; e abaixo, com os filitos
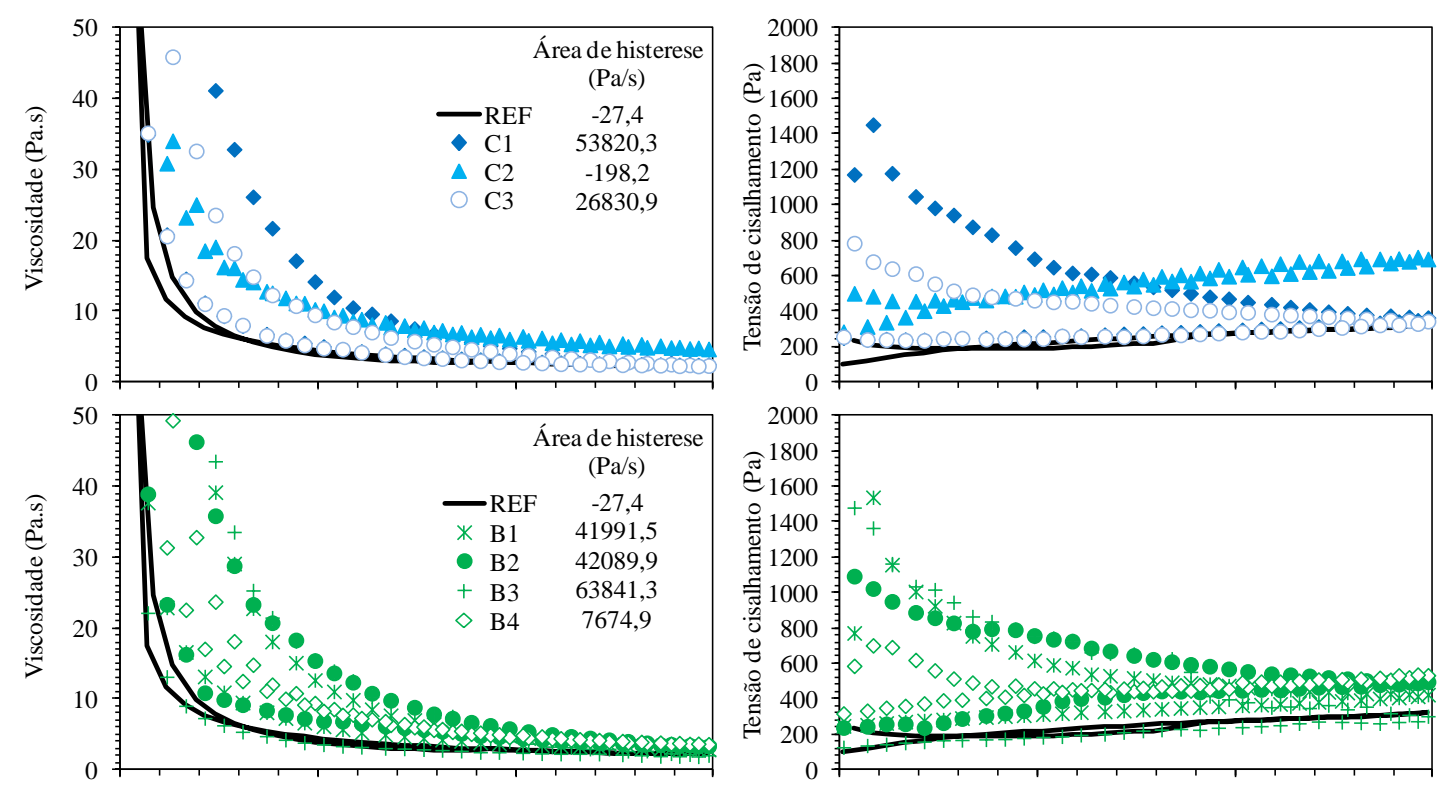

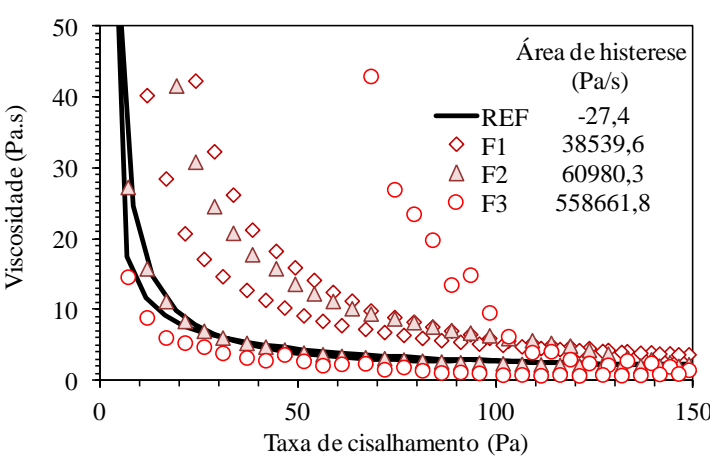

(a)

Após a aceleração, a taxa de cisalhamento foi reduzida até zero, possibilitando a ocorrência de uma reaglomeração das partículas, que foi dependente do tipo de material utilizado em substituição ao cimento.

No entanto, a tensão de cisalhamento foi menor que na etapa anterior, mostrando que a taxa de desaglomeração das partículas foi maior do que para a reaglomeração (perfil de tixotropia positiva).

Somente na pasta utilizada como referência não houve diferença entre a desaglomeração e a reaglomeração, ou seja, não foi gerada uma área de histerese considerável, e na pasta formulada com a cal C2 foi observado um perfil de tixotropia negativa (área de histerese negativa), ilustrando, diferentemente das demais, que a reaglomeração das partículas foi um pouco mais rápida do que a desaglomeração durante o aumento da taxa de cisalhamento imposta no ensaio.

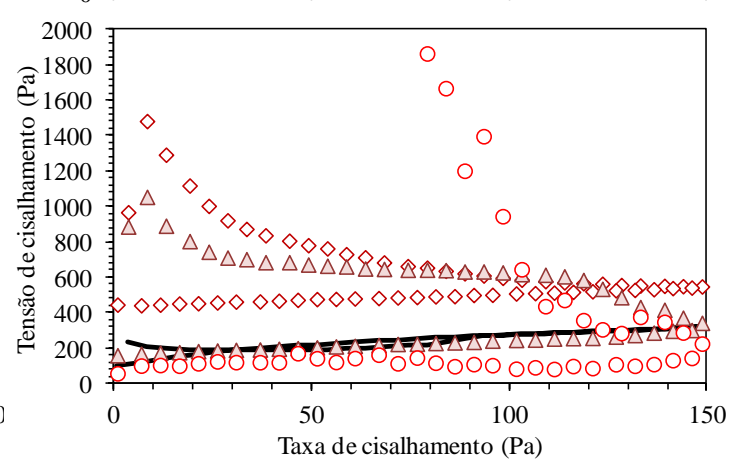

(b)

Hattori e Izumi (1982) explicam esse tipo de comportamento como uma competição entre os processos de coagulação e defloculação. Para essa teoria, o cimento está coagulando, mas essa coagulação pode ser reversível de acordo com a solicitação imposta ao material.

Como os argilominerais avaliados no trabalho são comercializados como insumos para utilização em argamassas em substituição à cal, e teoricamente conferem as mesmas características reológicas que os ligantes aéreos, os profissionais responsáveis pela execução da obra muitas vezes não percebem as diferenças durante o preparo e aplicação do revestimento. No entanto, podem ser observadas diferenças durante o endurecimento ou no desempenho do material em uso. 


\section{Cinética de aglomeração: reometria oscilatória}

A consolidação das pastas de cimento ocorre basicamente devido a dois fatores distintos, porém complementares: reação química de hidratação e coagulação/floculação (ROMANO; PILEGGI, 2012). Assim, um aumento no valor do módulo de armazenamento elástico (G') é esperado para esse tipo de material ao longo do tempo.

As forças de coagulação/floculação são intensificadas com o passar do tempo, devido à reação de hidratação, resultado do aumento da concentração iônica e da formação dos compostos hidratados do ligante. Esses fenômenos estão diretamente ligados à temperatura, pois a cinética de reação, o movimento browniano e a taxa de colisão entre as partículas são intensificados (ROMANO et al., 2011).
Na Figura 3 são apresentados os resultados de G' em função do tempo para as composições com cal (a), blenda (b) ou filito (c). A esquerda, são apresentados os gráficos conforme obtidos no ensaio, com G' variando em função da composição como um todo; e à direita, os valores de $G^{\prime}$ foram normalizados em função do teor de cimento nas composições, ou seja, em todos os casos assumiuse que $100 \%$ da composição seria o ligante hidráulico, visando melhor ilustrar o efeito das cales ou dos argilominerais durante a consolidação.

A deformação utilizada no ensaio ocorreu dentro do regime viscoelástico linear das pastas e, por isso, a estrutura gerada com a consolidação e hidratação não foi rompida durante o teste. Assim, foi possível quantificar a variação da tensão de escoamento ao longo do tempo (ROMANO et al., 2013).

Figura 3 - Cinética de aglomeração das pastas formuladas com cales (a), blendas (b) ou filitos (c)
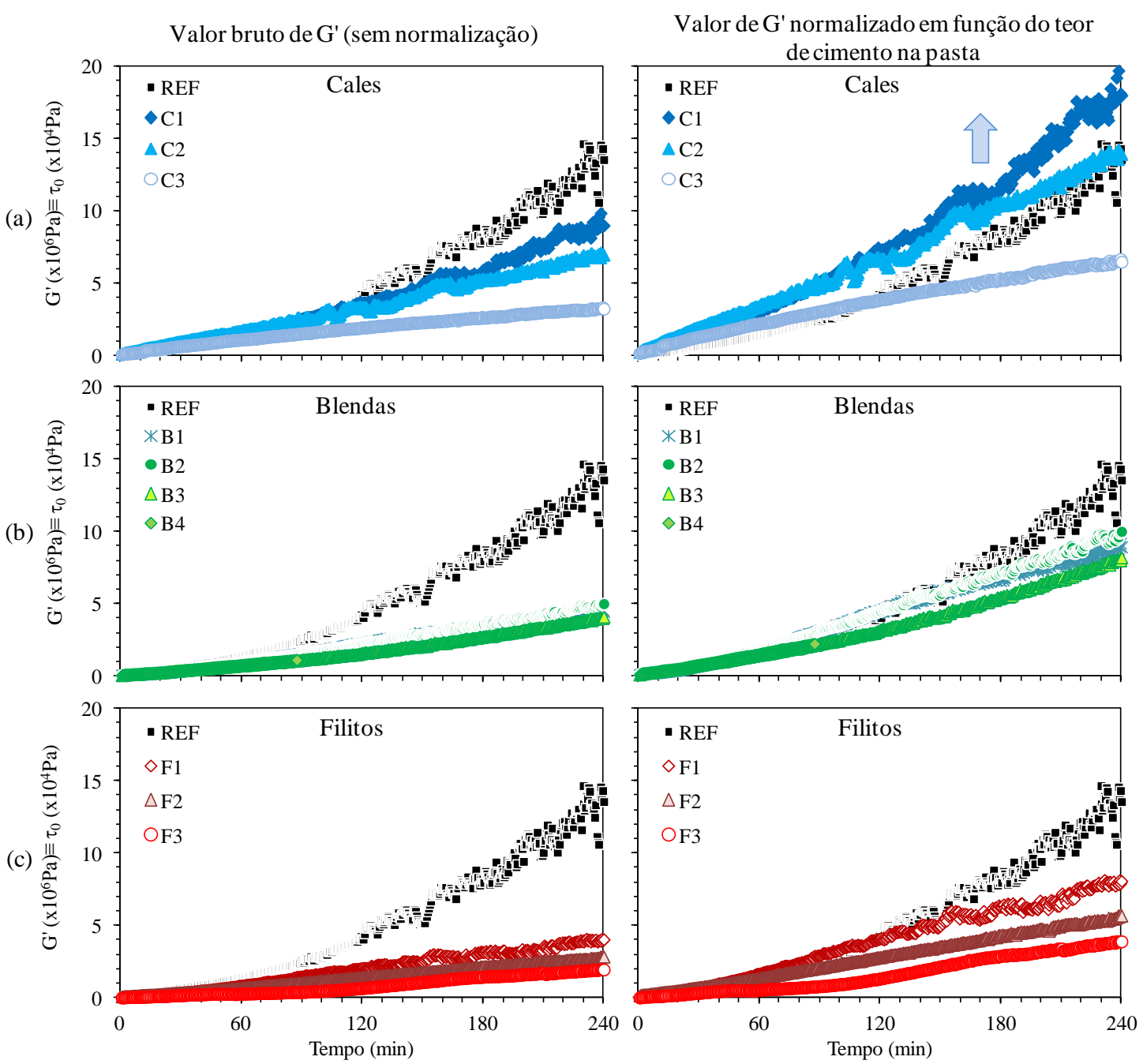

Nota: a esquerda, são apresentados os gráficos conforme obtidos no ensaio, com G' variando em função da composição como um todo, e à direita os valores de G' foram normalizados em função do teor de cimento. 
Nesta etapa, foi possível diferenciar as famílias de materiais onde as cales do tipo $\mathrm{CHI}$ ( $\mathrm{C} 1$ e $\mathrm{C} 2)$ apresentaram cinética de aglomeração mais acentuada do que nas demais pastas: a dissolução e precipitação dos hidratos aumentaram rapidamente a área superficial específica, pela formação do C$\mathrm{S}-\mathrm{H}$, e promoveram o aumento das forças de interação de van der Waals, diminuindo a distância de separação entre as partículas e aumentando a taxa de aglomeração (ROMANO et al., 2011, 2013).

Por outro lado, com a cal C3, dolomítica, o desenvolvimento da estrutura seguiu uma cinética de consolidação semelhante à das blendas e filitos. Esse fato é explicado, ao menos em parte, pelo fato de esta cal apresentar distribuição granulométrica mais aberta, menor área superficial específica e menor quantidade de $\mathrm{CaO}(\sim 39 \%)$ que as demais cales, fatores que poderiam reduzir a taxa de aglomeração. Além disso, trata-se de uma a cal do tipo CHIII, sendo mais de $30 \%$ de sua massa composta de filler calcário, material com menor solubilidade, enquanto as demais são tipo $\mathrm{CHI}$ e contêm menos de $12 \%$ de filler calcário.

As pastas formuladas com as blendas apresentaram cinéticas de consolidação mais acentuadas do que as pastas com os filitos puros, decorrente do maior teor de cal presente naquelas, o que confirma a maior capacidade aglomerante dos ligantes aéreos.

Essas observações estão diretamente relacionadas com a reação química de hidratação do cimento. Conforme reportado na literatura, os filitos não apresentaram nenhuma alteração na cinética de reação em comparação com a pasta de cimento, comprovando que esses minerais não apresentam nenhuma reatividade. Apesar disso, uma pequena diferença no nível de calor liberado em relação à pasta de cimento diz respeito à presença de maior quantidade de partículas que aumentaram a quantidade de pontos de nucleação para o início da cristalização dos compostos sólidos hidratados (ROMANO et al., 2011).

Sendo assim, como os filitos reconhecidamente não apresentam características ligantes, servem somente como inertes nas pastas cimentícias, e o desenvolvimento da microestrutura ocorre devido à ação do cimento.

As formulações com as cales do tipo CHI apresentam ganho de consistência mais acentuado do que as composições com os filitos, os quais mantêm as pastas com características viscosas por maior intervalo de tempo, ou seja, a tensão de escoamento sofre pouca influência durante o endurecimento das pastas com os filitos. Consequentemente, formulações com os argilominerais poderão necessitar maior tempo entre a aplicação e a realização do acabamento no revestimento aplicado.

\section{Conclusões}

Apesar do fato de as propriedades físicas, químicas e mineralógicas dos filitos serem distintas das propriedades das cales, as características das pastas durante o fluxo foram semelhantes, confirmando que, no caso da utilização desses materiais em substituição à cal nas composições de argamassas, não seriam notadas diferenças durante a aplicação do revestimento.

Durante o endurecimento, acompanhado a partir dos ensaios de reometria oscilatória, também não foram observadas consideráveis diferenças entre as pastas formuladas com os filitos, blendas e a cal do tipo CHIII.

Por outro lado, as cales do tipo CHI, representadas por $\mathrm{C} 1$ e $\mathrm{C} 2$, apresentaram taxa de aglomeração mais rápida que os demais materiais, fruto da maior área superficial específica e do maior teor de ligante na composição.

Essas características são importantes durante a execução do revestimento, porque, após a aplicação das argamassas, a tensão de escoamento deve aumentar rapidamente, para evitar o escorregamento da massa na parede. Além disso, o ganho de consistência deve ser rápido para não afetar de forma indesejada o tempo em aberto e, consequentemente, não diminuir a produtividade.

\section{Referências}

BANFILL, P. F. G. Rheology of Rresh Cement and Concrete. Edinburgh: School of the Built Environment, Heriot-Watt University, 2007. EH14 4AS.

FLATT, R. J. Dispersion Forces in Cement Suspensions. Cement Concrete Research, v. 34, n. 3, p. 399-408, 2004.

HATTORI, K.; IZUMI, K. A Rheological

Expression of Coagulation Rate Theory: parts 1-3. Journal of Dispersion Science Technology, v. 3, p. 129-193, 1982.

JOHN, V. M. O Papel da Cal na Longevidade dos Revestimentos. Revista Negócios da Cal, v. 26, n. 81, jun. 2003.

LIBERATO, C. C. et al. Efeito da Calcinação do Resíduo de Bauxita nas Características Reológicas e no Estado Endurecido de Suspensões Com Cimento Portland. Ambiente Construído, Porto Alegre, v. 12, n. 3, p. 7-23, jul./set. 2013. 
OLIVEIRA, I. R. et al. Dispersão e Empacotamento de Partículas: princípios e aplicações em processamento cerâmico. São Paulo: Fazendo Arte, 2000.

ROMANO, R. C. O. et al. Efeito da Presença de Cales e Filitos nas Propriedades de Pastas Imentícias. In: SIMPÓSIO BRASILEIRO DE TECNOLOGIA DE ARGAMASSAS, 9., Belo Horizonte, 2011. Anais... Belo Horizonte: ANTAC, 2011.

ROMANO, R. C. O; PILEGGI, R. G.

Temperature Influence on the Rheological Behavior of Cementitous Pastes Prepared With Air-Entraining Admixtures. Applied Rheology, n. 22, p. 20-28, 2012.

ROMANO, R. C. O. et al. Evaluation of Transition From fluid to Elastic Solid of Cementitious Pastes with Bauxite Residue Using Oscillation Rheometry and Isothermal Calorimetry. Applied Rheology, v. 23, p. 1-15, 2013.
ROUSSEL, N. et al. Steady State Flow of Cement Suspension: a micromechanical state of the art.

Cement Concrete Research, v. 40, n. 1, p. 77-84, 2010.

QUARCIONI, V. A.; CINCOTTO, M. A.

Influência da Cal Hidratada nas Idades Iniciais da Hidratação do Cimento Portland: estudo em pasta.. São Paulo, 2008. 20 p. Boletim Técnico da Escola Politécnica. BT/PCC/506

\section{Agradecimentos}

Os autores agradecem a FAPESP e a Associação Brasileira dos Produtores de Cal, pelo suporte durante a realização do trabalho.

\title{
Roberto Cesar de Oliveira Romano
}

Departamento de Construção Civil, Escola Politécnica | Universidade de São Paulo | Av. Professor Almeida Prado, Trav. 2, n. 83, Cidade Universitária | Caixa Postal 61548 | São Paulo - SP - Brasil | CEP 05424-970 | Tel.: (11) 3091-5248 | E-mail: rcorjau@gmail.com

\section{Mauro Adamo Seabra}

Associação Brasileira dos Produtores de Cal | Rua Marconi, 131, $11^{\circ}$ Andar, conjunto 1101 | São Paulo - SP - Brasil | CEP $01047-910$ | Tel.: (11) 3258-5366 | Email: mauroseabra@abpc.org.br

Vanderley Moacyr John

Departamento de Construção Civil, Escola Politécnica | Universidade de São Paulo | Tel.: (11) 3091-5794 | Email: vmjohn@usp.br

Rafael Giuliano Pileggi

Departamento de Construção Civil, Escola Politécnica | Universidade de São Paulo | Tel.: (11) 3091-5442 | Email: rafael.pileggi@usp.br

\author{
Revista Ambiente Construído \\ Associação Nacional de Tecnologia do Ambiente Construído \\ Av. Osvaldo Aranha, $99-3^{\circ}$ andar, Centro \\ Porto Alegre - RS - Brasil \\ CEP 90035-190 \\ Telefone: +55 (51) 3308-4084 \\ Fax: +55 (51) 3308-4054 \\ www.seer.ufrgs.br/ambienteconstruido \\ E-mail: ambienteconstruido@ufrgs.br
}

84 Romano, R. C. de O.; Seabra, M. A.; John, V. M.; Pileggi, R. G. 